\title{
Zivilgesellschaft als Legitimationsquelle in der asiatisch-europäischen Umwelt- und Klimakooperation
}

\section{Kurzfassung}

Die interregionale Umwelt- und Klimakooperation im Rahmen des Asia-Europe Meetings (ASEM) bietet den Mitgliedstaaten die Gelegenheit, vor dem Hintergrund stagnierender multilateraler Verhandlungen Problemlösung und Institutionalisierung auf einer untergeordneten Ebene zu betreiben. Interregionale Dialogprozesse bringen zwar keine verbindlich rechtlichen Regelungen hervor, können aber durchaus politische Entscheidungen auf regionaler und nationaler Ebene beeinflussen. Schon seit der Gründung von ASEM 1996 stand die Frage zivilgesellschaftlicher Partizipation und damit die der Legitimität dieses primär intergouvernementalen Prozesses im Raum. Diesbezügliche kulturelle Differenzen führten zur Gründung der Asia-Europe Foundation (ASEF) als sozio-kulturelle Säule des ASEM-Prozesses, in der die Zivilgesellschaft eine Dialogplattform fand. Gleichzeitig wurde damit zivilgesellschaftliche Partizipation vom politischen Dialog entkoppelt. Die Agenda von ASEF wurde durch die Organisationsstruktur der Stiftung von ASEM-Mitgliedstaaten mitbestimmt. Betrachtet man die Partizipationsmöglichkeiten in ASEM genauer, stellt sich die Frage, welchen Beitrag zivilgesellschaftliche Akteure zur Legitimität des Prozesses leisten können. Einerseits zeigten die Aktivitäten des Asia-Europe Environment Forum (ENVforum) unter zivilgesellschaftlicher Beteiligung bereits Wirkung auf die ASEM-Gipfel und fördern insgesamt den Informations- und Wissenstransfer. Andererseits kann die partizipierende Zivilgesellschaft als entpolitisiert betrachtet werden und Legitimitätskriterien wie Inklusion, Konsultation, Transparenz oder Respons zeigen sehr gemischte Ergebnisse. 


\section{Inhalt}

1. Einleitung: ASEM und die Frage nach der Legitimität 518

a) Warum zivilgesellschaftliche Partizipation im ASEM-Prozess? 519

b) Wer partizipiert? Politisierte und entpolitisierte Zivilgesellschaft im ASEM-Prozess

c) Legitimität in der Interregionalismusforschung 521

d) Gliederung des Beitrags und methodisches Vorgehen 522

2. Legitimität interregionaler Beziehungen durch zivilgesellschaftliche Akteure?

3. Der ASEM-Prozess und zivilgesellschaftliche Partizipation in ASEF 526

4. Partizipation und Konsensbildung in den umweltpolitischen Aktivitäten von ASEF

a) Inklusion und Konsultation 531

b) Transparenz

c) Konsensbildung: Interaktion zwischen ENVforum und ASEMMitgliedern

5. Fazit: Legitimität durch zivilgesellschaftliche Partizipation?

\section{Einleitung: ASEM und die Frage nach der Legitimität}

Im Kontext vermehrt stagnierender multilateraler Verhandlungen werden politische Entscheidungsprozesse zunehmend auf sub-globale Ebenen verlagert. Inzwischen gibt es in der derzeitigen Global Governance-Architektur zwischen der multilateralen und nationalen Ebene zahlreiche Dialogforen, die nach oben und nach unten politisch, ökonomisch, sozial und ökologisch Einfluss ausüben können. Ein Beispiel für solch eine zwischengeschaltete Ebene stellen die europäisch-asiatischen Beziehungen als interregionale Kooperationsform dar.

Einhergehend mit diesem Phänomen stellt sich die Frage nach der demokratischen Qualität und Legitimität solcher Prozesse, welche in der politikwissenschaftlichen Forschung oft im Rahmen der von Scharpf (1999) etablierten Kategorien der Inputund Output-Legitimität diskutiert wird. Der vorliegende Beitrag setzt sich ebenfalls mit dieser zentralen Fragestellung auseinander und analysiert am Beispiel des AsiaEurope Meetings (ASEM), welche Gestaltungs- und Einflussmöglichkeiten zivilgesellschaftliche Akteure, verstanden als non-profit-Akteure, im ASEM-Prozess haben oder, anders ausgedrückt, welchen Beitrag diese zu dessen Input-Legitimität im Sinne von Partizipation und Konsensbildung leisten können. Diese Fragestellung 
wird im Folgenden dahingehend eingegrenzt, inwiefern die integrierte zivilgesellschaftliche Partizipation im Kontext der umweltpolitischen Aktivitäten der AsiaEurope Foundation (ASEF) zur Input-Legitimität des ASEM-Prozesses beiträgt.

\section{a) Warum zivilgesellschaftliche Partizipation im ASEM-Prozess?}

ASEM gilt als eines der ältesten und am stärksten institutionalisierten interregionalen Dialogforen. Singapur und Frankreich lancierten 1994 den Vorschlag, einen regelmäßigen Gipfel zwischen Asien und der Europäischen Union (EU) zu organisieren. Knapp zwei Jahre später wurde ASEM bei dem ersten Gipfel in Bangkok als ein ökonomisches, politisches und kulturelles Dialogforum etabliert. Die Gründung von ASEM fällt in eine Zeit stagnierender multilateraler, aber auch interregionaler Verhandlungen zwischen der EU und der Association of Southeast Asian Nations (ASEAN) (Gaens 2008 a: 9 ff.; University of Helsinki Network for European Studies 2006: $4 \mathrm{ff}$.) und gleichzeitig proliferierender Freihandelsabkommen rund um das Pazifische Becken. ASEM sollte eine Plattform außerhalb existierender multilateraler Institutionen bieten, mit dem vorrangigen Ziel, die ökonomische Integration beider Regionen voranzutreiben (Gaens 2008 b: 29; Maull 2010: 183-185), zugleich aber auch Kooperation in anderen Bereichen wie zum Beispiel Kultur oder Bildung zu ermöglichen. So kamen beispielsweise auch Umweltthemen auf die politische Agenda, die im ASEM-Prozess durch das ASEM Environment Ministerial Meeting sowie das Asia-Europe Environment Forum (ENVforum) institutionell verankert wurden. Daneben erschien ASEM insbesondere für die Europäer geeignet, um einen besseren Zugang zum asiatischen Markt zu bekommen und eine zunehmende Präsenz der USA im asiatisch-pazifischen Raum auszugleichen (Hwee 2007: 185).

Ein kritischer Punkt bei der Gründung von ASEM war der Stellenwert zivilgesellschaftlicher Partizipation. Diese Frage erscheint in der internationalen Politik von besonderer Relevanz, da zivilgesellschaftlichen Akteuren eine Scharnierfunktion zwischen den Bürgern und politischen Entscheidungsprozessen auf internationaler Ebene zugesprochen wird (Steffek u. a. 2010: 103). Diese ist beispielsweise bei der Formulierung und Umsetzung von Nachhaltigkeitszielen, wie sie auch im ASEM-Prozess artikuliert werden, von Bedeutung, da diese in Einklang mit Prioritäten regionaler, nationaler und lokaler Akteure gebracht werden sollten (Auer 2000: 174). Entscheidend ist, dass die Interessen und Bedürfnisse beispielsweise von Umweltproblemen betroffener Personen in politische Prozesse eingebracht werden. Das Potenzial von beispielsweise Nicht-Regierungs-Organisationen (NGOs), verstanden als "non-state, non-profit, non-violent organizations with the 
main objective of pursuing political and/or social change without striving for governmental power" (Beyer 2007: 514), liegt neben ihrer fachspezifischen Expertise insbesondere darin, die Distanz zwischen politischen Entscheidungsträgern und den betroffenen Akteuren zu reduzieren und politische Entscheidungen in lokale Kontexte einzubetten. Ihnen wird somit attestiert, dass sie durch entsprechende Beteiligung am politischen Prozess die Effizienz und Legitimität internationaler Entscheidungsfindungsprozesse erhöhen (ebd.). Die Frage nach der Legitimität interregionaler Dialogprozesse, die letztendlich keine rechtlich verbindlichen Regeln hervorbringen, ist deshalb relevant, weil auf der einen Seite interregionale Prozesse wie ASEM durchaus politische Entscheidungen auf regionaler und nationaler Ebene beeinflussen können; auf der anderen Seite bieten diese aber auch ein Forum, um multilaterale Verhandlungen zu ergänzen oder ggf. zu umgehen und auf einer untergeordneten Verhandlungsebene Probleme zu lösen und neue Institutionen zu schaffen. Gerade vor dem Hintergrund der zunehmenden institutionellen Fragmentierung der internationalen Umwelt- und Klimapolitik (Biermann u. a. 2009; Oberthür/Stokke 2010; Zelli 2011) leistet die Betrachtung interregionaler Umweltkooperation und die Legitimität interregionaler Beziehungen einen signifikanten Beitrag zu aktuellen Forschungsfragen der internationalen Umwelt-Governance.

\section{b) Wer partizipiert? Politisierte und entpolitisierte Zivilgesellschaft im AS- EM-Prozess}

ASEM ist ein Hybrid hinsichtlich zivilgesellschaftlicher Partizipation, denn man kann sowohl von einer integrierten, in Form der Asia-Europe Foundation (ASEF), als auch von einer oppositionellen Beteiligung, in Form des Asia-Europe People's Forum (AEPF), sprechen. Dies lässt sich damit erklären, dass die Aufnahme der Zivilgesellschaft als einer der Hauptakteure in das Asia-Europe Cooperation Framework (AECF) hauptsächlich auf Druck der EU geschah (Bersick 2008: 246). ${ }^{1}$ Der zivilgesellschaftliche Dialog wurde daraufhin durch die Gründung von ASEF im Jahr 1997 institutionalisiert. ASEF bietet eine Plattform für zivilgesellschaftliche Interaktion mit dem Ziel, "to promote better mutual understanding between Asia and Europe through greater intellectual, cultural and people exchanges" (ASEM 2005). Bersick weist darauf hin, dass ASEF der Rechtsform nach zwar eigenständig, allerdings nach wie vor eine intergouvernementale Organisation sei. ASEF wird von den ASEM-Mitgliedern finanziert. Das Agenda Setting und die Frage, welche zivilgesellschaftlichen Organisationen partizipieren können bzw. dürfen, werde durch

1 Siehe auch Asia-Europe Cooperation Framework (AECF) 2000, http://eeas.europa.eu/asem/docs/ aecf_2000_en.pdf(Stand: 14.10.2013). 
die einzelnen Regierungen über das Board of Governors beeinflusst (Bersick 2008: 247). Das AEPF kritisierte die starke Dominanz ökonomischer Themen im ASEMProzess, die eingeschränkten zivilgesellschaftlichen Beteiligungsmöglichkeiten (auch im Hinblick auf ASEF) und teilweise auch die Finanzierungspraktiken von ASEF, die kleinere NGOs benachteilige (Keva 2008: 110). Entsprechend dieser Auffassung hieße das, dass ASEF als integraler Bestandteil des ASEM-Prozesses primär wenig umstrittenen und entpolitisierten zivilgesellschaftlichen Akteuren Gehör verschaffe. Das AEPF bildete sich daher 1996 bewusst als eine transnationale Oppositionsbewegung heraus, welches parallel zu den ASEM-Gipfeltreffen organisiert wird und einer kritischen, politisierten Zivilgesellschaft eine Plattform bietet, um die Agenda des alternativen Regionalismus voranzutreiben. ${ }^{2}$ Auch wenn es inzwischen etwas mehr Berührungspunkte zwischen dem AEPF und ASEM-Vertretern gibt, wie insbesondere der ASEM6-Gipfel demonstrierte (ebd.: 112), bestehen die zwei Dimensionen zivilgesellschaftlichen Engagements - Integration und Opposition - im ASEM-Prozess fort (Zajak 2012: 3).

\section{c) Legitimität in der Interregionalismusforschung}

Zur Beantwortung der Fragestellung greift der vorliegende Beitrag auf die Legitimitätsbegriffe und -kriterien von Scharpf (1999), Dryzek (2000), Bernstein (2004), Dingwerth (2007), Bäckstrand u. a. (2010 b), Steffek/Hahn (2010), Steffek u. a. (2010), Steffek/Nanz/Kissling (2008) und Schmidt (2010) zurück. Gleichzeitig wird diese Thematik in den Kontext der Interregionalismusforschung gestellt. ${ }^{3}$ Dieser Forschungszweig hat sich bereits intensiv mit den Funktionen des Interregionalismus und ASEM als entsprechend transregionaler Kooperationsform auseinandergesetzt. Aufgrund der Exekutivlastigkeit interregionaler Zusammenarbeit wird dabei immer wieder auf das damit einhergehende Demokratiedefizit verwiesen (Rüland 2014: 19). Die damit zusammenhängende Frage nach der Legitimität solcher Prozesse wird verstärkt mit dem Aspekt zivilgesellschaftlicher Beteiligung verknüpft (Rüland 2010: 1276). Studien von Grugel (2004), Gilson (2005), Bersick (2008), Keva (2008), Manea (2008) oder Zajak (2012) setzen sich unter anderem mit dieser Thematik auseinander. Meist geht es dabei allerdings um die grundsätzlichen institutionellen Beteiligungsmöglichkeiten zivilgesellschaftlicher Akteure

2 Siehe hierzu auch die Charter des Asia-Europe People's Forum, http://www.tni.org/archives/act/ 18722, sowie http://www.alternative-regionalisms.org/wp-content/uploads/2009/09/asia-europepeoples-forum-peoples-response-to-asem-2000.pdf (Stand: 5.3.2014).

3 Vergleiche Robles 2008; Hänggi/Roloff/Rüland 2006; Hänggi 2006; Roloff 2006; Rüland 2002, 2006, 2010, 2012, 2014; Gilson 2002, 2005. 
und Fallbeispiele konzentrieren sich weitestgehend auf das Themenfeld Menschenrechte. Diesbezügliche Analysen zur ASEM Umwelt- und Klimakooperation existieren beispielsweise nicht. Der vorliegende Artikel wird daher mit dem Ziel verfolgt, den Demokratisierungs- bzw. Legitimitätsaspekt interregionaler Beziehungen am Beispiel des ASEM Environment Forums (ENVforum) zu diskutieren.

\section{d) Gliederung des Beitrags und methodisches Vorgehen}

Der Beitrag gliedert sich wie folgt: In einem ersten Schritt werden die Legitimitätskriterien unter Einbezug der bisherigen Erkenntnisse der Interregionalismusforschung kurz dargestellt. Basierend auf diesen Erkenntnissen werden anschließend die institutionellen Grundlagen des ASEM-Prozesses sowie zivilgesellschaftlicher Partizipation als Beitrag zur Input-Legitimität anhand der umweltpolitischen Arbeit von ASEF diskutiert. Es wird sich dabei zeigen, dass ASEF durch diverse Aktivitäten zivilgesellschaftlichen Akteuren eine Gesprächsplattform bietet und Austausch mit Regierungsvertretern ermöglichen kann. Zugleich wird jedoch auch am Beispiel der Umweltkooperation deutlich werden, dass die Interaktion zwischen zivilgesellschaftlichen und politischen Akteuren innerhalb des ASEM-Prozesses stark entpolitisiert und stets kontextabhängig ist. ${ }^{4}$

Grundsätzlich sollte die Frage nach zivilgesellschaftlicher Beteiligung sowohl im Kontext der Input- als auch Output-Legitimität betrachtet werden. Denn mit einem zunehmenden Einfluss interregionaler Aushandlungsprozesse auf die Institutionalisierung umweltpolitischer Kooperation oder beispielsweise nationale Politikformulierung und Entscheidungsfindungsprozesse stellt sich vermehrt die Frage nach umfassenden Beteiligungsmöglichkeiten auf übergeordneten Ebenen. Da bereits seit den 1990er Jahren ein Demokratiedefizit bei ASEM konstatiert und kritisiert wird, welches zu einer graduellen Öffnung zivilgesellschaftlicher Partizipation geführt hat, konzentriert sich dieser Beitrag zunächst auf die Input-Legitimität. In weiterführenden Studien sollte explizit auch auf die Output-Dimension eingegangen werden.

Methodisch basiert die Analyse auf einem deduktiven Vorgehen, das heißt die zuvor erarbeiteten Legitimitätskriterien werden am genannten Fallbeispiel erörtert. Die Untersuchung stützt sich dabei auf eine eingehende Dokumentenanalyse von Primär- und Sekundärquellen wie beispielsweise ASEM-Abschlussdokumente, von ASEF veröffentlichte Berichte und Studien, Publikationen von NGOs sowie wissenschaftliche Analysen. Ergänzt werden diese Quellen durch qualitative, semi-

4 Zum Vergleich siehe beispielsweise die Analyse von Manea (2008) zur Menschenrechtskooperation und Zajak (2012) zu Arbeitsrechten. 
Zivilgesellschaft als Legitimationsquelle

strukturierte Interviews ${ }^{5}$ mit an der ASEF-Umweltkooperation beteiligten Akteuren.

\section{Legitimität interregionaler Beziehungen durch zivilgesellschaftliche Ak- teure?}

Gerade in der internationalen Umwelt- und Klimapolitik sind zahlreiche beteiligte Akteure enttäuscht über langatmige und ineffektive Verhandlungen, mangelnde Kompromissfähigkeit, Inflexibilität, institutionelle Pfadabhängigkeiten und realitätsferne Entscheidungen. Die Frage nach der Problemlösungskapazität verlagert sich daher verstärkt auf die sub-globalen Ebenen. Regionale, nationale und insbesondere lokale Ebenen erscheinen besser geeignet, holistische Politikansätze zu entwickeln und flexibler auf jegliche Art von Veränderungen zu reagieren.

Im Mittelpunkt bisheriger Interregionalismus-Forschung standen daher vorwiegend Fragen nach der Akteursqualität der beteiligten Regionalorganisation bzw. Staaten sowie den Funktionen, die diese Ebene in der Global Governance-Architektur erfüllen kann (Rüland 2014: 17). Die meisten Analysen ${ }^{6}$ beschäftigten sich daher mit diesen Funktionen: (soft) balancing (regionaler Machtausgleich), multilateral utility (Agenda-Setting, Institutionenbildung, Ausarbeitung gemeinsamer Positionen für multilaterale Verhandlungen), collective identity-building (Ausbildung regionaler Identitäten). Neuere Studien befassen sich mit dem Aspekt der Norm- und Politikdiffusion durch interregionale Kooperation (Grugel 2007; Manea 2008; Carrapatoso 2011), welcher allerdings nach wie vor weiterführender Forschung bedarf (Rüland 2014: 27). Fragen nach der Demokratisierung von und durch interregionale Kooperation werden in der bisherigen Interregionalismusforschung immer wieder in Studien eingeflochten, insbesondere im Hinblick auf zivilgesellschaftliche Beteiligung. ${ }^{7}$ Die Frage nach dem Beitrag zivilgesellschaftlicher Akteure zur Legitimierung interregionaler Prozesse wird dabei oft nur implizit betrachtet und nicht systematisch analysiert. Geht man davon aus, dass demokratische Impulse aus interregionalen Beziehungen herrühren können, wie beispielsweise von Pevehouse (2002), Mansfield und Pevehouse (2006) oder Keohane, Macedo und Moravcsik (2009) für internationale Organisationen, Regime und Netzwerke diskutiert wird, so sollte die demokratische Qualität dieser Beziehungen selbst sowie die Frage nach deren Legitimität untersucht werden.

5 Die Interviews wurden sowohl persönlich per Telefon als auch per E-Mail durchgeführt.

6 Siehe bspw. Dent 2004; Hänggi/Roloff/Rüland 2006; Robles 2008; Rüland 2010; Rüland u. a. 2008.

7 Vergleiche Grugel 2004; Gilson 2005; Bersick 2008; Keva 2008; Manea 2008; Zajak 2012. 
Fritz Scharpf (1999) trennt den Begriff der Legitimität idealtypisch in eine Inputund eine Output-Dimension. Input-Legitimität fragt nach Partizipation und Konsensbildung in politischen Entscheidungsprozessen, Output-Legitimität befasst sich mit dem Aspekt der Effektivität und Problemlösungskapazität. Zivilgesellschaftliche Akteure wie NGOs werden bzgl. des Partizipationskriteriums oft als Allheilmittel gesehen, um internationale Entscheidungsfindungsprozesse demokratischer zu gestalten und somit einen Beitrag zur Legitimität internationaler Abkommen zu leisten. Durch ihre aktive Teilnahme an globalen Gestaltungs- und Regulierungsprozessen wird ihnen die Rolle legitimer und legitimierender Akteure zugesprochen, die die Effizienz und Legitimität internationaler Entscheidungsfindungsprozesse erhöhen (Beyer 2007: 514). Doch allein durch ihre Sichtbarkeit und die Aufmerksamkeit, die ihnen seitens der Politik geschenkt wird, sind weder die Wichtigkeit von NGOs und ihr Einfluss auf Politikergebnisse (Williams 2005: 345) noch die Gewährleistung demokratischer Prozesse belegt. ${ }^{8}$ Steffek u. a. (2010) befassen sich ebenfalls mit der demokratischen Legitimität von zivilgesellschaftlichen Organisationen und nennen diesbezüglich Inklusion, Transparenz, Partizipation, Unabhängigkeit und Respons in (politischen) Entscheidungsprozessen als entscheidende Kriterien. Daraus folgend müssen sie selbst Input- und Output-Legitimität in ihren internen Prozessen gewährleisten. ${ }^{9}$ Als legitime Akteure werden NGOs und andere Formen zivilgesellschaftlichen Engagements dann auch zu legitimierenden Akteuren in den internationalen Beziehungen.

Im ASEM-Prozess muss es zunächst einmal als Fortschritt angesehen werden, dass in einem intergouvernemental angelegten und konsequenterweise exekutivlastigen Dialogprozess überhaupt zivilgesellschaftliche Akteure eingebunden werden. Daher geht es im vorliegenden Beitrag nicht darum zu analysieren, ob diese Akteure legitim sind, sondern inwiefern diejenigen, die beteiligt sind, einen Beitrag zur Legitimität des gesamten Prozesses leisten können. Da bei ASEM die Deliberation viel stärker als der politische Output im Sinne von Effektivität und Problemlösungskapazität im Vordergrund steht, wird im folgenden der Fokus auf die InputLegitimität nach Scharpf (1999) gelegt und mit den analytischen Überlegungen von Dingwerth (2007) zu demokratischer Legitimität verbunden. Die Aspekte Partizipation und Konsensbildung stehen dabei als zentrale Analysekriterien im Vorder-

8 Zum Demokratisierungspotenzial und Legitimität von NGOs in den internationalen Beziehungen siehe auch die kritischen Analysen von Brand 2005, Altvater/Brunnengräber 2000 oder Beisheim 2005.

9 Siehe hierzu auch Atack (1999) über die vier Legitimitätskriterien von Entwicklungshilfeorganisationen. Zu den einzelnen Funktionen von NGOs siehe beispielsweise Frantz/Martens 2006; Doherty 2002. 
grund. Da der Dialog im ASEM-Prozess und insbesondere in der Arbeit von ASEF stärker im Fokus ist als der eigentliche politische Entscheidungsprozess, wurde für die vorliegende Untersuchung das Analyseraster etwas vereinfacht. So unterscheidet Dingwerth zwischen Partizipation und Inklusion (Bandbreite und Qualität), demokratischer Kontrolle (Verantwortlichkeit und Transparenz) und deliberativer Praxis (Universalität, Rationalität, Reziprozität) (ebd.: $27 \mathrm{ff}$.). Diese wurden aufgrund der empirischen Überlappung unter den zwei oben genannten Hauptkriterien subsumiert. Ergänzt werden diese durch von Steffek u. a. (2010), Bäckstrand u. a. (2010 a), Kronsell/Bäckstrand (2010) und Schmidt (2010) weiter ausdifferenzierte Aspekte. Abbildung 1 spiegelt die genannten Kriterien der Input- bzw. demokratischen Legitimität und die für die Operationalisierung des Ansatzes zentralen Analysefragen wider.

Im vorliegenden Beitrag wird allerdings der Aspekt der Konsensbildung viel stärker mit der Frage nach der Interaktion zwischen dem ENVforum und den ASEM-Mitgliedern verbunden. Da es innerhalb des ENVforums primär um den Dialog zwischen den beteiligten Akteuren und weniger um die Etablierung eines politischen Konsenses geht, tritt die Frage nach Konsensbildung im ENVforum in den Hintergrund. Relevanter ist die Frage, wie die Ergebnisse und Anregungen des ENVforum

Abbildung 1: Legitimitätskriterien in Deliberationsprozessen

\begin{tabular}{|c|c|c|c|c|c|}
\hline \multicolumn{6}{|c|}{ Input-/Demokratische Legitimität } \\
\hline \multicolumn{3}{|c|}{ Partizipation } & \multicolumn{3}{|c|}{ Konsensbildung } \\
\hline Inklusion & Konsultation & Transparenz & $\begin{array}{c}\text { Formelle } \\
\text { Regeln }\end{array}$ & $\begin{array}{l}\text { Informelle } \\
\text { Praxis }\end{array}$ & Respons \\
\hline \multicolumn{3}{|c|}{$\begin{array}{l}\text { - Wer nimmt am politischen } \\
\text { Entscheidungsfindungsprozess teil? Welche Reichweite } \\
\text { und Qualität besitzt die Inklusion? } \\
\text { - Wie werden relevante und von politischen } \\
\text { Entscheidungen betroffene Akteure ausgewählt, } \\
\text { identifiziert und im politischen Prozess repräsentiert? } \\
\text { Wie transparent ist das Auswahlverfahren? } \\
\text { - Wie offen oder geschlossen ist die Partizipation, d.h. } \\
\text { sind die Partizipationsmöglichkeiten und -bedingungen } \\
\text { für alle betroffenen Akteure gleich? } \\
\text { - Wie sieht die institutionelle Ausgestaltung des } \\
\text { Deliberationsprozesses aus? } \\
\text { Wie werden Transparenz und Zugang zu Informationen } \\
\text { und somit die Zuordnung von Verantwortlichkeit } \\
\text { gewährleistet? }\end{array}$} & \multicolumn{3}{|c|}{$\begin{array}{l}\text { - Wie werden politische Entscheidungen getroffen? } \\
\text { - Welche formellen und informellen Regeln und } \\
\text { Praktiken existieren? } \\
\text { Zu welchem Zeitpunkt nehmen welche Akteure mit } \\
\text { welcher Gewichtung teil? Welche Argumente von } \\
\text { welchen Akteuren werden berücksichtigt? } \\
\text { - Wie transparent ist der Entscheidungsprozess? } \\
\text { - Wie werden Ergebnisse des politischen } \\
\text { Entscheidungsprozesses nach innen und nach } \\
\text { außen kommuniziert? } \\
\text { - Wie zeichnet sich der Deliberationsprozess in } \\
\text { politischen Entscheidungen ab? }\end{array}$} \\
\hline
\end{tabular}

Quelle: Eigene Darstellung. 
formell oder informell die Regierungsvertreter erreichen und ein entsprechender Respons im Kontext der ASEM-Gipfel zu verzeichnen ist. Dies wird der Schwerpunkt bei der Betrachtung des Aspekts Konsensbildung darstellen.

Bäckstrand u. a. (2010 b: 17 f.) merken kritisch an, dass Partizipation nicht automatisch die Performanz politischer Entscheidungen erhöhe. Zudem führe die Dominanz marktliberaler Akteure in deliberativen Prozessen zu Machtasymmetrien. Letztlich sei auch eine grundlegende Ungleichheit in Bezug auf die Partizipationsmöglichkeiten der verschiedenen Akteure zu verzeichnen. Diese Kritik kann man durchaus auf den ASEM-Prozess übertragen. Umso mehr stellt sich die Frage, ob die Einbindung zivilgesellschaftlicher Akteure tatsächlich zur Legitimität des Prozesses beiträgt oder ob die Partizipation rein symbolischer Natur ist. Dies wird am Beispiel der umweltpolitischen Aktivitäten in den folgenden Kapiteln diskutiert.

\section{Der ASEM-Prozess und zivilgesellschaftliche Partizipation in ASEF}

Der ASEM-Prozess basiert auf drei Säulen mit entsprechenden Themenschwerpunkten in den jeweiligen institutionalisierten Deliberationsplattformen (siehe Abb. 2). Die Interaktion der verschiedenen beteiligten Akteure basiert auf Informalität, Multi-Dimensionalität und gleichwertiger Partnerschaft beider Regionen. Diese Partnerschaft soll sowohl durch Aktivitäten auf höchster politischer Ebene als auch auf zivilgesellschaftlicher Ebene gefestigt werden. ${ }^{10}$ Das ASEM-Gipfeltreffen ist das höchste Entscheidungsgremium und bestimmt weitestgehend die inhaltliche Ausrichtung des Prozesses. Hier sowie in Treffen zwischen den Außenministern und leitenden Regierungsmitgliedern findet der politische Dialog statt. Die Aktivitäten und Initiativen der anderen Säulen sollen inhaltlich diesen Dialog aufgreifen und unterstützen. ${ }^{11}$

10 Die Publikation der Europäischen Kommission (COM1.1, 1994) "Towards a New Strategy for Asia”, bestätigt einen Wandel in den asiatisch-europäischen Beziehungen. Siehe auch ASEM History, http://www.aseminfoboard.org/index.php?option=com_k2\&view=item\&layout=item \&id=823\&Itemid=236 (Stand: 9.3.2013).

11 Siehe ASEM Working Methods, http://www.aseminfoboard.org/working-method.html (Stand: 11.7.2013). 


\begin{tabular}{|c|c|c|c|}
\hline \multicolumn{4}{|c|}{$\begin{array}{c}\text { ASEM Gipfeltreffen } \\
\text { Regierungschefs, Staatsoberhäupter, Präsident der Europäischen Union }\end{array}$} \\
\hline & Politisch & Ökonomisch & Sozio-kulturell \\
\hline Akteure & Regierungschefs, Minister & $\begin{array}{l}\text { Minister, privater/ } \\
\text { öffentlicher Sektor }\end{array}$ & $\begin{array}{l}\text { Regierungschefs, Minister, } \\
\text { Zivilgesellschaft }\end{array}$ \\
\hline $\begin{array}{l}\text { Thematische } \\
\text { Schwerpunkte }\end{array}$ & $\begin{array}{l}\text { Internationale Krisen } \\
\text { Sicherheit } \\
\text { Multilateralismus } \\
\text { Sektorale Dialoge (Umwelt, } \\
\text { Menschenrechte, Migration) }\end{array}$ & $\begin{array}{l}\text { Globalisierung } \\
\text { Nachhaltige Entwicklung } \\
\text { Handelserleichterungen/ } \\
\text { Handelssicherheit } \\
\text { Handel und Entwicklung } \\
\text { Regionalismus/ } \\
\text { Multilateralismus } \\
\text { Informations- und } \\
\text { Kommunikationstechnologie } \\
\text { Investitionen }\end{array}$ & $\begin{array}{l}\text { Kultur } \\
\text { Bildung } \\
\text { Thematische } \\
\text { Ausdifferenzierung in ASEF } \\
\text { (Wirtschaft\&Gesellschaft, } \\
\text { Nachhaltige } \\
\text { Entwicklung\&Umwelt, } \\
\text { Wissenschaftliche } \\
\text { Kooperation\&Bildung, } \\
\text { Kunst\&Kultur, } \\
\text { Governance\&Menschenrech } \\
\text { te, Gesundheit) }\end{array}$ \\
\hline Institutionen & $\begin{array}{l}\text { Politische High-Level-Dialoge } \\
\text { Reguläre (Außen-) } \\
\text { Ministertreffen } \\
\text { Asia-Europe Parliamentary } \\
\text { Partnership (ASEP) }\end{array}$ & $\begin{array}{l}\text { Reguläre Ministertreffen } \\
\text { ASEM Trust Fund } \\
\text { Asia-Europe Business Forum } \\
\text { (AEBF) } \\
\text { European Financial Expertise } \\
\text { Network (EFEX) }\end{array}$ & $\begin{array}{l}\text { Asia-Europe Foundation } \\
\text { (ASEF) } \\
\text { Asia-Europe Higher } \\
\text { Education exchange } \\
\text { ASEM Education Secretariat } \\
\text { Trans-Eurasian Information }\end{array}$ \\
\hline
\end{tabular}

Quelle: Eigene Darstellung.

Wie Abbildung 2 verdeutlicht, ist am politischen Entscheidungsfindungsprozess aufgrund der dreigliedrigen Struktur und des stark ausgeprägten intergouvernementalen Charakters von ASEM zunächst einmal nur die Teilnahme von Nationalstaaten vorgesehen. So wird die politische Agenda durch die in der Regel alle zwei Jahre stattfindenden Gipfeltreffen bestimmt. Im politischen Dialog sind vorwiegend Regierungsmitglieder vertreten. Seit dem Jahr 2006 kamen durch die Asia-Europe Parliamentary Partnership (ASEP) Parlamentarier aus beiden Regionen hinzu, die allerdings über keinerlei Entscheidungskompetenzen verfügen. ${ }^{12}$ Zivilgesellschaftliche Akteure partizipieren fast ausschließlich in der sozio-kulturellen Säule im

12 Siehe hierzu auch eine kurze Notiz des Parliament of Finland, Asia-Europe Parliamentary Partnership Meeting, http://web.eduskunta.fi/Resource.phx/parliament/internationalorgans/ finnishdelegationtotheasia-europeparliamentarypartnershipmeeting.htx; ASEM, Asia-Europe Parliamentary Partnership Meeting, http://www.aseminfoboard.org/asia-europe-parliamentary-part nership-meeting-asep.html (Stand: 16.9.2013). 
Rahmen von ASEF-Aktivitäten. Durch die Zusammensetzung der einzelnen Foren innerhalb von ASEF, der Organisationsstruktur der Stiftung und säulenübergreifenden Interaktionsmechanismen wie den Ad-hoc-Treffen gibt es durchaus Berührungspunkte mit Regierungsvertretern. Wie gewichtig diese Interaktion letztendlich für den politischen Entscheidungsprozess ist, muss an einzelnen Fallbeispielen diskutiert werden, da davon ausgegangen werden muss, dass diese Gewichtung je nach Thema und Zeitpunkt unterschiedlich bewertet werden kann.

Das Mandat von ASEF bezieht sich auf den intellektuellen und kulturellen Austausch, um das gegenseitige Verständnis der Regionen zu verbessern. Als interkulturelle Austauschplattform hat ASEF das Ziel, interregionale Netzwerke und Kooperation zu befördern sowie Erfahrungsaustausch und ein Lernen durch Dialog zu ermöglichen (ASEF 2011 a). Im Prinzip 2 der Dublin Principles werden ASEF's Aufgaben festgelegt (ASEM 2005):

,a) organizing projects closely linked with ASEM activities in collaboration with relevant Asian and European institutions of the ASEM countries;

b) implementing any project assigned by future meetings of ASEM leaders or Ministers and the relevant member of the European Commission;

c) acting as a clearing house, catalyst and facilitator;

d) organizing a few flagship projects of its own;

e) conducting public relations activities to profile ASEM, and publicize ASEM meetings, seminars and other activities; and

f) giving grants."

An diesen Aufgaben wird deutlich, dass ASEF von vornherein lediglich Raum für die Partizipation einer entpolitisierten Zivilgesellschaft bietet und Beteiligungsmöglichkeiten aufgrund der Gründungslogik von ASEM begrenzt sind (siehe Kapitel 1).

Grundsätzlich werden Deliberationsprozesse in verschiedenen Foren nach dem Prinzip der Multi-Akteurs-Konstellationen organisiert. So nehmen in den verschiedenen Dialogforen staatliche Akteure, Vertreter der Europäischen Kommission (EK) und des Sekretariats der Association of Southeast Asian Nations (ASEAN), epistemische Gemeinschaften, Nicht-Regierungs-Organisationen (NGOs) und Vertreter der Privatwirtschaft teil. ${ }^{13}$ Laut den in den Dublin Principles formulierten Zielen stehen ASEF-Aktivitäten grundsätzlich im Einklang mit den Interessen der Mitgliedstaaten. Daher können von der Stiftung besetzte Themen als unkontrovers bezeichnet werden. Demnach kann ASEF verstärkt als Implementierungsinstanz

13 Für eine Auflistung der Partner und Sponsoren von ASEF siehe http://www.asef.org/index.php/ about/partners\# (Stand: 21.2.2014). 
denn als gleichwertiger Partner bei der Entscheidungsfindung gesehen werden. Trotz dieser Rollenverteilung ist die Beziehung zwischen den Aktivitäten von ASEF und deren Einfluss auf die ASEM-Gipfeltreffen und damit auf den politischen Dia$\log$ nicht immer klar. Denn teilweise erscheint beispielsweise das ENVforum auch als Impulsgeber für eine Umwelt- bzw. Nachhaltigkeitsagenda in der interregionalen Kooperation, wie der ASEM6-Gipfel demonstrierte (siehe Kapitel 4). Dies hängt, wie im folgenden Kapitel erläutert werden wird, mit den unterschiedlichen Partizipationsmöglichkeiten von ASEF zusammen. Denn teils interagieren die einzelnen Säulen durch Ad-hoc-Treffen formell, ein bedeutender Teil der Interaktion verläuft allerdings informell. Die Berührungsflächen zwischen ASEF-Akteuren und ASEM-Mitgliedern sind oft indirekter Natur durch die Beteiligung nationalstaatlicher Vertreterinnen und Vertreter an den ASEF-Foren.

\section{Partizipation und Konsensbildung in den umweltpolitischen Aktivitäten von ASEF}

Im Bereich der Umwelt-Governance versucht ASEF, verschiedene zivilgesellschaftliche Akteure in Gesprächsforen, Runde Tische, Konferenzen und Workshops einzubeziehen. Das ASEF Umweltprogramm kann in die Bereiche Bildung, Kultur und Politik unterteilt werden. Im Rahmen der Bildung spielen sowohl der wissenschaftliche Austausch zwischen Experten als auch Umweltbildungsprogramme für Jugendliche und junge Erwachsene eine Rolle. Zu nennen sind hier die ASEF University, das Asia-Europe Forestry Exchange Programme oder gezielte Veranstaltungen im Rahmen existierender Programme wie das 9. Asia-Europe Classroom Network (AEC-NET). ${ }^{14}$

Der umweltpolitische Dialog zwischen politischen und zivilgesellschaftlichen Akteuren im Kontext von ASEM wurde insbesondere seit 2003 durch das von ASEF initiierte Environment Forum (ENVforum) institutionalisiert. Das ENVforum bietet eine Plattform für den Austausch von Ideen, Normen und die Gestaltung bestimmter umwelt- und klimarelevanter Politikbereiche, aber auch für Agenda Setting im asiatisch-europäischen Dialogprozess und die Bildung weiterer Institutionen, insbesondere innerhalb der ASEF. Der erste in diesem Zusammenhang organisierte Runde Tisch fand 2003 in Bangkok statt. 41 Vertreter der Wissenschaft, des Privatsektors,

14 Für die letzte an Umweltthemen orientierte ASEF University siehe http://www.asef.org/index.php/ projects/themes/environment/2491-AU18 (Stand: 31.7.2013); für einen Verweis auf die Programme 2001-2003 siehe Asia-Europe Forestry Exchange Programme, http://www.asef.org/index.php/ projects/programmes/575-asia-europe-forestry-experts-exchange-programme (Stand: 31.8 .2012 ); für Informationen zum 9. AEC-NET siehe http://www.asef.org/index.php/projects/themes/en vironment/645-9th-aec-net_conference (Stand: 31.7.2013). 
lokaler Regierungen, NGOs sowie Mitglieder der Europäischen Kommission, der Asian Development Bank (ADB), der Europäischen Umweltagentur sowie des ASEAN Sekretariats nahmen hieran teil (ASEF 2011 b). Das ENVforum wurde bewusst ins Leben gerufen, um zivilgesellschaftlichen Akteuren aus beiden Regionen eine Dialogplattform sowie Gelegenheiten zur Netzwerkbildung und zum Austausch von Expertenwissen zu bieten. ${ }^{15}$ Seit dem Gründungsjahr organisiert ASEF das Forum gemeinsam mit der Hanns-Seidel-Stiftung (Deutschland), dem Institute for Global Environmental Strategies (IGES, Japan) sowie dem United Nations Environment Programme (UNEP). Seit 2006 beteiligt sich das Swedish Environmental Secretariat for Asia (SENSA) der Swedish International Development Cooperation Agency (SIDA) an der Organisation. ${ }^{16}$ Das Steering Committee (SC), welches die Agenda des ENVforum festlegt, besteht in geringfügig wechselnden Konstellationen aus intergouvernementalen/nationalen Akteuren und Vertretern epistemischer Gemeinschaften. ${ }^{17}$ Projekte und Themen, die vom ENVforum bearbeitet werden, müssen vom sogenannten Board of Governors (bestehend aus den ASEM-Mitgliedsländern ohne zivilgesellschaftliche Beteiligung), welches sich alle zwei Jahre trifft, bewilligt werden. So wird auch die Projektfinanzierung gesichert. ${ }^{18}$ Betrachtet man die zu diskutierenden Themen, so fällt auf, dass diese sich besonders auf internationale Herausforderungen wie etwa im Kontext der internationalen Klimaverhandlungen oder Rio +20 beziehen. Es ist zudem zu vermuten, dass aufgrund der Bewilligung seitens des Board of Governors keine für die jeweiligen Mitgliedsländer kontroversen Themen auf die Agenda gesetzt werden.

15 E-Mail-Korrespondenz mit einem ehemaligen Mitglied des Steering Committees im September 2012.

16 Informationen aus einer Informationsbroschüre des ENVforums vom Mai 2010 zum Thema „An Asia-Europe Platform for Multistakeholder Dialogue and Debate“ sowie E-Mail-Korrespondenz mit einer ASEF-Mitarbeiterin im März 2014.

17 So sind und waren dies das ASEAN-Sekretariat, die Asian Development Bank (ADB), die Europäische Umweltagentur (EEA), die Europäische Kommission, das United Nations Environment Programm (UNEP), die Swedish International Development Cooperation Agency (SIDA), das Swedish Environmental Secretariat (SENSA), das Hamburg Institute of International Economics, das Regional Institute of Environmental Technology, die Chinese Academy of Social Sciences, das Energy and Resources Institute (TERI), das Institute for Global Environmental Strategies (IGES), die Hanns-Seidel-Stiftung, das Regional Environment Center, der Earth Council, KEHATI - The Indonesian Biodiversity Foundation; siehe dazu Fort 2005 sowie ASEF, "1 1 th $^{\text {th }}$ Asia Europe Environment Forum (ENVforum) Steering Committee Meeting”, http://www.asef.org/index.php/ projects/themes/environment/630-11 th-asia-europe-environment-forum-envforum-steerin-comm ittee-meeting (Stand: 29.7.2013); ASEF, "Asia Europe Environment Forum (ENVforum): Asia Europe Strategies for Earth Summit 2012”, http://www.asef.org/images/docs/ENVforum 2011_concept.pdf (Stand: 29.7.2013).

18 Persönliches Telefoninterview mit einer ASEF-Mitarbeiterin im Oktober 2012. 


\section{a) Inklusion und Konsultation}

Die involvierten NGOs können grundsätzlich als eher unkontrovers bezeichnet werden. So geht beispielsweise das Regional Environment Center auf eine Initiative der USA, der Europäischen Kommission und Ungarns zurück und unterstützt schwerpunktmäßig Länder aus Ost- und Südosteuropa in umweltpolitischen Themen. ${ }^{19}$ Der Earth Council Asia-Pacific ist eine NGO im Kontext der Agenda 21 und somit eng mit UNEP verknüpft. ${ }^{20}$ Die KEHATI Stiftung wurde in ihrer zehnjährigen Gründungsphase (1995-2005) basierend auf einem Kooperationsabkommen von USAID, der US Entwicklungsbehörde, finanziell unterstützt. Sie agierte jedoch stets als unabhängige NGO und wurde in einer Evaluation im Jahre 2005 als eine der führenden indonesischen NGOs im Bereich Biodiversität bezeichnet (Paget u. a. 2005). The Energy and Resources Institute (TERI) in Indien hat seine Ursprünge in einer Unternehmensinitiative und verbindet somit eine NGO mit dem Privatsektor. ${ }^{21}$

Grundsätzlich steht die Partizipation im Rahmen von ASEF allen interessierten zivilgesellschaftlichen Akteuren offen. Es gibt öffentliche Einladungen zur Mitarbeit, die regelmäßig auf der Website publiziert und etwa über den Newsletter des International Institute for Sustainable Development (IISD) verteilt werden. Wird seitens einer Organisation oder einer Person Interesse an der Mitarbeit signalisiert, wird die Relevanz und Vereinbarkeit mit der Agenda überprüft und ggf. eine Einladung verschickt. Zudem verfügt ASEF über ein internes Netzwerk sowie das ASEF Alumni Netzwerk, so dass bereits existierende Kooperationspartner oder ggf. neue Teilnehmer je nach Thema direkt kontaktiert werden. Die SC-Mitglieder werden ebenfalls um Empfehlungen gebeten, sobald das Thema einer Veranstaltung feststeht, da davon ausgegangen wird, dass diese über ihre jeweiligen Netzwerke über relevante Kontakte verfügen. Es ist bei der Auswahl besonders wichtig, dass möglichst viele der Mitgliedsländer vertreten sind. Gerade bei den politischen Dialogen ist die Rekrutierung über bereits bestehende Netzwerke eine gängige Praxis, da hier Politikempfehlungen verfasst werden und dementsprechend gegenseitiges

19 Für weitere Informationen siehe Regional Environment Center, http://www.rec.org (Stand: 4.9.2012).

20 Für weitere Informationen siehe Earth Council Asia-Pacific, http://www.environmental-main streaming.org/Partner\%20Organisations/po_ecap.html und http://ekh.unep.org/?q=node/52 (Stand: 4.9.2012).

21 Für mehr Informationen zu TERI siehe auch The Energy and Resources Institute, "About TERI", http://www.teriin.org/index.php?option=com_content\&task=view\&id=36 (Stand: 4.9.2012). 
Vertrauen eine große Rolle spielt. Persönliche Vorschläge bzgl. potenzieller Teilnehmer haben daher ein besonderes Gewicht. ${ }^{22}$

An den umwelt- und klimapolitischen Veranstaltungen von ASEF nimmt regelmäßig ein kleiner Kreis von Akteuren teil. ASEF sucht je nach thematischem Schwerpunkt zusätzliche Experten. ${ }^{23}$ Diese werden direkt angesprochen und zu Workshops, Foren etc. eingeladen. Da sich das ENVforum zum Ziel gesetzt hat, komplementär zu existierenden Prozessen zu arbeiten, sind die zu behandelnden Themen oft sehr spezifisch. Laut ASEF ist es daher eine Herausforderung, die entsprechenden Experten zu akquirieren. Als Beispiel kann hier die Spezialisierung auf „Institutional Framework for Sustainable Development" genannt werden, die sich an der Arbeit von UNEP orientiert. Entsprechend wurde UNEP bzgl. möglicher Kooperationspartner für das ENVforum konsultiert, da UNEP über ein entsprechendes Netzwerk verfügt. Gleiches gilt für den zweiten wichtigen Partner des ENVforum, die Hanns-Seidel-Stiftung, die ebenfalls über ein umfassendes Partnernetzwerk, gerade in der asiatischen Region, verfügt. Insgesamt greift ASEF auf ein sehr breites Expertennetzwerk zurück. Diese Einladungspraxis, insbesondere für den politischen Dialog, lässt darauf schließen, dass die Teilnehmer aufgrund ihrer thematischen Expertise und bereits existierender Kooperation entweder mit ASEF oder den Partnerorganisationen (z. B. UNEP, Hanns-Seidel-Stiftung) sowie persönlicher Empfehlungen ausgewählt werden. ${ }^{24}$

\section{b) Transparenz}

Zum Thema Transparenz und öffentlicher Zugang zu Informationen lässt sich festhalten, dass die Ergebnisse der Workshops, Konferenzen und Dialogforen nur bedingt umfassend dokumentiert bzw. zugänglich sind. Diese werden oft in Form von Sammelbänden veröffentlicht, die größtenteils nicht auf der Website frei verfügbar sind und über ASEF direkt bestellt werden müssen. ${ }^{25}$ Bei laufenden Prozessen bzw. politikberatenden Aktivitäten zeigt sich ein etwas anderes Bild. Jüngstes Beispiel für ein politikrelevantes Treffen ist die Strategieentwicklung für Rio+20. Hier wurde

22 Persönliches Telefoninterview mit einer ASEF-Mitarbeiterin im Oktober 2012 sowie nachfolgende E-Mail-Korrespondenz; E-Mail-Korrespondenz mit einem ehemaligen SC-Mitglied im September 2012.

23 E-Mail-Korrespondenz mit der Hanns-Seidel-Stiftung im Januar 2014; persönliches Telefoninterview mit einer ASEF-Mitarbeiterin im Oktober 2012.

24 Persönliches Telefoninterview mit einer ASEF-Mitarbeiterin im Oktober 2012 sowie nachfolgende E-Mail-Korrespondenz; E-Mail-Korrespondenz mit einem ehemaligen SC-Mitglied im September 2012.

25 Beispiele für eine ausführliche Dokumentation sind folgende Sammelbände: Molina (2005), Fort (2004), Fort/Iglesias (2006). 
nur begrenzt Material öffentlich zur Verfügung gestellt. So sind Ziele und die diskutierten Themen klar. Jedoch wurde auf eine der breiten Öffentlichkeit zugängliche Zusammenfassung der Ergebnisse bislang verzichtet. ${ }^{26}$ Dies kann zwar einerseits auf ein Ressourcenproblem zurückzuführen sein; andererseits ließe es sich auch dahingehend interpretieren, dass dies aufgrund der Sensibilität politischer Dialogprozesse zunächst nicht beabsichtigt war.

Nach wie vor versuchen zivilgesellschaftliche Akteure gemeinsame Positionen zum Thema nachhaltige Entwicklung bzw. den Sustainable Development Goals (SDGs) zu erarbeiten und diese den ASEM-Mitgliedern zu unterbreiten. Im Kontext der Vor- und Nachbereitung von Rio +20 beteiligte sich ASEF an einer großen Umfrage zu den SDGs. Dieses Projekt wurde vom Stakeholder Forum for a Sustainable Future $^{27}$ initiiert und wird vom deutschen Rat für Nachhaltige Entwicklung ${ }^{28}$ unterstützt. Durch das eingerichtete SDG e-Inventory ${ }^{29}$ als Online-Dialogplattform werden Vorschläge zu den SDGs von allen interessierten Akteure aus Zivilgesellschaft, Wirtschaft und intergouvernementalen Organisationen gesammelt und anschließend zusammengefasst. Beteiligt sind auch 14 weitere zivilgesellschaftliche Partner, die nicht nur öffentliche Aufmerksamkeit auf das Projekt lenken, sondern auch verschiedene regionale Aspekte einbringen, Kapazitäten aufbauen und Informationen für zivilgesellschaftliche Aufklärung verbreiten sollen. ASEF kümmert sich um die diesbezügliche Verbreitung in Asien. Eine Zusammenfassung und alle Beiträge sind frei zugänglich. ${ }^{30}$ Im Februar 2014 wurde in diesem Zusammenhang der „Small Planet Report“ veröffentlicht. ${ }^{31}$

26 Zugängliches Material findet sich unter ASEF, "Strategies for the Earth Summit 2012", http://ww w.asef.org/index.php/projects/themes/environment/1998-strategies-for-the-earth-summit-2012-\# 2549-asia-europe-strategies-for-earth-summit-2012; siehe hierzu auch kurze Informationen zum III. Scenario Planning Workshop of the Asia Europe Environmental Forum in Bangkok, http://w ww.hss.de/southeastasia/en/indonesia/news-events/2012/hsf-jakarta-as-a-member-of-the-steering -committee-for-the-iii-scenario-planning-workshop-of-the-asia-europe-environmental-forum-inbangkok.html (Stand: 26.7.2013).

27 Für mehr Informationen über das Forum siehe http://www.stakeholderforum.org/sf/ (Stand: 10.10.2013).

28 Siehe hierzu auch http://www.nachhaltigkeitsrat.de (Stand: 10.10.2013).

29 Siehe hierzu auch http://www.sdgseinventory.org/ (Stand: 10.10.2013).

30 E-Mail-Korrespondenz mit einer ASEF-Mitarbeiterin im Juli 2013.

31 Für die elektronische Version des Berichts siehe http://www.asef.org/images/docs/Small_Pla net_20311213_clean.pdf (Stand: 21.2.2014). 


\section{c) Konsensbildung: Interaktion zwischen ENVforum und ASEM-Mitgliedern}

Neben den Partizipationsmöglichkeiten und -bedingungen sowie Transparenz spielt für die deliberative Qualität auch der Zugang zu den Entscheidungsträgern eine wichtige Rolle, das heißt welchen Einfluss können die beteiligten zivilgesellschaftlichen Akteure auf die ASEM-Mitgliedstaaten ausüben. Eine indirekte Verbindung lässt sich durch die Teilnahme eines Mitarbeiters des ASEAN-Sekretariats an der Arbeit des ENVforums vermuten. Das ASEAN-Sekretariat ist gleichzeitig auch an den ASEM-Gipfeln beteiligt. Der Kontakt zum ASEAN-Sekretariat ist in den letzten Jahren insgesamt intensiver geworden. Dieser wird unter anderem durch Veranstaltungen wie den „Workshop on Sustainable Development Goals (SDGs) for ASEAN countries“ “ im September 2013 gefördert. ${ }^{32}$ Der enge Bezug zwischen dem ENVforum und ASEAN wird insbesondere durch regelmäßige Kontakte mit dem Leiter des „Departments for Environment and Disaster Mitigation“ des ASEANSekretariats aufrecht erhalten..$^{33}$ Kontakte zur Europäischen Union hingegen sind eher sporadischer Natur. Hier ist insbesondere ein leitender Mitarbeiter der Europäischen Umweltagentur Ansprechpartner. Vertreter der Europäischen Kommission nehmen nicht am ENVforum teil. Allerdings geht es dem ENVforum um eine personenorientierte und weniger eine institutionalisierte Einbindung. ${ }^{34}$

Neben einer Rezeption der Arbeit des ENVforums auf regionaler Ebene stoßen die Ergebnisse ebenfalls auf der UN-Ebene auf Resonanz. Hier existieren enge Kontakte zum Leiter des Bereichs „Strategic Resource Mobilization and Special Initiatives" von UNEP. So wurden die Ergebnisse des ENVforums auf allen hochrangigen UN-Konferenzen zu nachhaltiger Entwicklung vorgestellt. Beide Kontakte sind gleichzeitig auch SC-Mitglieder von ASEF. ${ }^{35}$ Bereits frühere Initiativen wie die Konferenz in Jakarta zum Thema „1/3 of Our Planet: What Can Asia and Europe do for Sustainable Development?" 36 zeigten Wirkung auf globaler Ebene. So ist laut UNEP die Harmonisierung der Basel-, Rotterdam- und Stockholm-Konventionen im Kontext der „11 th Special Session of the UNEP Governing Council/Global Ministerial Environment Forum" bzw. der "Simultaneous Extraordinary Meetings of the Conferences of the Parties to the Basel, Rotterdam and Stockholm Conventions" unter anderem auf die Ergebnisse der Jakarta Konferenz des ENVforums zurück-

32 Für mehr Informationen siehe http://www.asef.org/index.php/projects/themes/sustainable-deve lopment/3012-workshop-on-sustainable-development-goals-sdgs-for-asean-countries (Stand: 21.2.2014).

33 E-Mail-Korrespondenz mit der Hanns-Seidel-Stiftung im Februar 2014.

34 E-Mail-Korrespondenz mit der Hanns-Seidel-Stiftung im Februar 2014.

35 E-Mail-Korrespondenz mit der Hanns-Seidel-Stiftung im Februar 2014.

36 Siehe dazu auch Fort/Iglesias 2006. 
zuführen. Diese Konferenz wurde seinerzeit auch von dem damaligen Direktor von UNEP, Klaus Töpfer, besucht. ${ }^{37}$ Gleichzeitig beeinflusste die Jakarta-Konferenz auch die schwedische bzw. SIDAs Asienstrategie und führte dazu, dass SENSA seit 2006 das ENVforum aktiv unterstützt. ${ }^{38}$

Die ASEM-Mitgliedstaaten direkt zu erreichen, erweist sich in der Praxis oft als schwierig. Hier agieren die für die Organisation des ENVforums zuständigen ASEFMitarbeiter als Transmissionsriemen. Diese nutzen Möglichkeiten der Kontaktaufnahme insbesondere über die nationalen Umweltministerien und ASEM-Kontaktstellen. Zusätzlich bieten große multilaterale Verhandlungen, wie beispielsweise Rio+20, Gelegenheiten zur Kontaktaufnahme und Interaktion. ASEF verteilte hier beispielsweise die Ergebnisse der umweltpolitischen Arbeit der Stiftung an relevante Regierungsvertreter. Am erfolgversprechendsten scheinen jedoch der ASEMGipfel selbst und die ASEM Senior Officials Meetings zu sein. ASEF organisiert in diesem Kontext Informationsveranstaltungen. Damit die Informationen allerdings entsprechend in den ASEM-Gipfel integriert werden, braucht ASEF die Unterstützung seitens der Regierungen. Entsprechend werden diejenigen Regierungsvertreter kontaktiert, für die die umweltpolitischen Themen von besonderer Relevanz sein könnten. Auf diese Weise können umwelt- bzw. klimapolitische Fragen wie Umwelt- und Energiesicherheit, nachhaltige Energie ${ }^{39}$ oder Nachhaltigkeitsziele $^{40}$ auf der ASEM-Agenda, aber auch regionalen bzw. nationalen Agenden gehalten werden. Der Einfluss auf die richtungsweisenden Abschlussdokumente der ASEM-Gipfel muss jedoch als relativ gering bewertet werden.$^{41}$ Grundsätzlich kann jedoch festgehalten werden, dass die Initiativen und Ergebnisse des ENVforums im Rahmen der ASEM-Treffen vorgestellt und verabschiedet werden, ${ }^{42}$ somit also die Arbeit des ENVforums von den ASEM-Mitgliedern zur Kenntnis genommen und zu einem gewissen Grad berücksichtigt wird.

Dass die Arbeit des ENVforums einen gewissen Einfluss auf die ASEM-Gipfeltreffen in Umweltthemen hatte und hat, kann zusätzlich in zeitlicher Dimension

37 Weitere Informationen zu der UNEP-Konferenz unter http://www.unep.org/gc/gcss-xi/ (Stand: 5.3.2014); E-Mail-Korrespondenz mit einer ASEF-Mitarbeiterin im März 2014.

38 E-Mail-Korrespondenz mit einer ASEF-Mitarbeiterin im März 2014.

39 Siehe dazu auch die "Chairman's Press Remarks 3 - Sustainable energy including environment and energy security",

http://www.asem6.fi/NEWS_AND_DOCUMENTS/EN_GB/1157973421290/INDEX.HTM (Stand: 5.3.2014).

40 Siehe dazu beispielsweise der Workshop für ASEAN-Länder zu diesem Thema, http://www.asef. org/index.php/projects/themes/environment/3012-workshop-on-sustainable-development-goalssdgs-for-asean-countries (Stand: 5.3.2014).

41 Persönliches Telefoninterview mit einer ASEF-Mitarbeiterin im Oktober 2012.

42 E-Mail-Korrespondenz mit der Hanns-Seidel-Stiftung im Februar 2014. 
festgemacht werden. Das ENVforum wurde 2003 gegründet. Im Rahmen des ASEM6 Gipfels im Jahre 2006 wurde das Thema nachhaltige Entwicklung und somit Umweltfragen offiziell in die interregionale Agenda integriert und Inhalte wurden seitdem durch die Gipfeltreffen zunehmend erweitert (siehe z. B. ASEM 2006 a). Die von ASEF initiierten umweltbezogenen Aktivitäten zeigen daher eine gewisse Wirkung - zumal dies offiziell im Chairman's Statement genannt wird. So betont das ASEM6 Chairman's Statement: ,[T] hey [ASEM member states] also noted the valuable role of related activities including the ASEM Oceans Initiative and the Asia Europe Environment Forum“ (ASEM 2006 b, Absatz 27). Beim ASEM7-Gipfel wurde ebenfalls Bezug auf das ENVforum genommen: „Leaders acknowledged the key message on sustainable energy from the 6th Asia-Europe Environment Forum" (ASEM 2008, Absatz 30). Diese offizielle Anerkennung der Arbeit des ENVforums zeigt, dass ASEF als offizielle Plattform für zivilgesellschaftliche Kooperation in einem begrenzten Maße zur Input- Legitimität der ASEM-Gipfeltreffen beiträgt.

Insbesondere ASEM6 und ASEM7 haben verdeutlicht, dass die Interaktion der am ENVforum beteiligten zivilgesellschaftlichen Akteure im Agenda Setting zumindest eine gewisse Wirkung zeigt. Allerdings wurde in den Abschlusserklärungen von ASEM8 und ASEM9 nicht mehr explizit Bezug auf die Arbeit des ENVforum genommen (ASEM 2010; ASEM 2012). Die Erklärungen gehen durchaus auf Umweltthemen und Klimaschutz ein, orientieren sich jedoch im Wesentlichen an internationalen Entwicklungen und Verhandlungsergebnissen der Klima- und Umweltkonferenzen der Vereinten Nationen (VN). Erwähnt werden beispielsweise auch Ministertreffen oder von einzelnen Ländern wie Japan oder Korea initiierte Workshops oder Foren, jedoch keine konkreten Aktivitäten des ENVforums.

ASEM8 verabschiedete einen Anhang, in dem Aktivitäten für die Jahre 2010-2012 gelistet wurden. ${ }^{43}$ Daraus entstanden sind zwei Institutionen, die explizit den Wissens- und Technologietransfer zwischen Europa und Asien fördern sollen. Erstens wurde das ASEM SMEs Eco Innovation Centre (ASEIC) in Korea im Februar 2011 gegründet. Dessen Ziel ist es, Wissenstransfer und die Verbreitung ökologischer Geschäftsideen und grüner Technologien zu unterstützen sowie kleineren und mittleren Unternehmen diesbezüglich Beratung anzubieten. ASEIC soll insbesondere als Dialog- und Netzwerkplattform dienen. Die Organisation von Konferenzen und Workshops, die Etablierung globaler Partnerschaften sowie die Kooperation mit internationalen Organisationen zu diesen Themen bilden die Aufgaben-

43 Für eine Themenliste siehe ASEM8 Chair's Statement - Annex I: List of new initiatives, http:// www.aseminfoboard.org/summit-statement.html (Stand: 29.7.2013). 
schwerpunkte von ASEIC. ${ }^{44}$ Zweitens wurde ebenfalls im Kontext des ASEM8Gipfels die Idee des ASEM Water Resources Research and Development Centers ${ }^{45}$ geboren. Dieses Zentrum wurde offiziell 2011 in China gegründet, um Wissens- und Technologietransfer zwischen Asien und Europa im Bereich des Wassermanagements zu fördern. Die neun aufgeführten Forschungsprojekte, die 2011 begonnen wurden, beziehen sich auf die Themen nachhaltige Wasserwirtschaft, Abwasser und Biodiversität in China. Daran angelehnt sind auch die Publikationen des Zentrums. Seit 2011 wurden keine neuen Projekte mehr gelistet. Es bleibt abzuwarten, ob es sich bei dem Zentrum um ein chinesisches Prestigeobjekt mit einem entsprechenden geografischen Forschungsschwerpunkt handelt oder ob sich die interregionale Forschungszusammenarbeit in der Zukunft auf weitere Länder erstreckt.

\section{Fazit: Legitimität durch zivilgesellschaftliche Partizipation?}

Das Beispiel des ASEF ENVforums als Teil der sozio-kulturellen Säule des ASEMProzesses hat gezeigt, dass zivilgesellschaftliche Akteure einen Beitrag zur InputLegitimität des ASEM-Prozesses leisten können. Betrachtet man Input-Legitimität unter den Aspekten Partizipation und Konsensbildung, Letzteres verstanden als die Interaktionsmöglichkeiten von Zivilgesellschaft und ASEM-Mitgliedern, fällt dieser Beitrag jedoch eher gering aus.

- Partizipation: Durch den engen Bezug zu den ASEM-Mitgliedern über das Board of Governors und die Gründungslogik von ASEF wird der Aufgabenbereich und die Agenda des ENV forums zumindest indirekt von Regierungsseite beeinflusst. Es ist daher wenig überraschend, dass von vornherein eher unkritische bzw. entpolitisierte zivilgesellschaftliche Akteure am ENVforum und vor allem am Steering Committee (SC) beteiligt sind. Es lässt sich auch eine besonders hohe Beteiligung epistemischer Gemeinschaften konstatieren. Grundsätzlich steht jeder zivilgesellschaftlichen Organisation die Teilnahme am ENVforum offen. Jedoch sollte unterschieden werden zwischen dem SC und den restlichen Aktivitäten des ENVforum. Foren, Workshops, Konferenzen etc. werden öffentlich bekannt gegeben und über verschiedene Medien beworben. Gleichzeitig werden mögliche Experten direkt eingeladen, die meist über ein bereits existierendes Netzwerk innerhalb von ASEF rekrutiert werden. Die Praxis hat gezeigt, dass meist ein relativ kleiner Kreis von zivilgesellschaftlichen Akteuren an den Aktivitäten des ENVforums teilnimmt. Das

44 Für weitere Informationen siehe http://www.aseic.org/main.do, http://www.aseminfoboard.org/ asem-smes-eco-innovation-center-aseic.html (Stand: 29.7.2013).

45 Für weitere Informationen siehe http://www.asemwater.org (Stand: 29.7.2013). 
$\mathrm{SC}$ ist ein geschlossener Kreis. Das Auswahlverfahren dieser Akteure ist für AuBenstehende eher intransparent und die Selektion beruht sehr stark auf Mund-zuMund-Propaganda. Es ist bezeichnend, dass die Zusammensetzung seit der Gründung des ENVforums weitestgehend konstant geblieben ist.

- Konsensbildung: Zivilgesellschaftliche Akteure sind im ENVforum beteiligt, jedoch nicht am politischen Dialog von ASEM. Es gibt keine formellen Regeln, die die Beziehung zwischen dem ENVforum und den anderen Säulen des ASEM-Prozesses bzw. den ASEM-Gipfeltreffen institutionalisieren. Vielmehr sind es die informellen Praktiken, die insbesondere seitens ASEF selbst genutzt werden, die diesen Kontakt herstellen, um Informationen sowohl weiterzuleiten als auch zu generieren. Dies gilt auch für die Beziehungen zu Regionalorganisationen wie ASEAN oder der globalen Ebene wie der UN, die insbesondere auf die enge personelle Verzahnung von ASEF und diesen Organisationen zurückzuführen ist. Formell geregelt ist lediglich das Verhältnis zwischen dem SC und dem Board of Governors in Bezug auf das Agenda Setting des ENVforums. Somit wird eine gewisse Interessenkongruenz zwischen den ASEM-Mitgliedern und beteiligten Akteuren im ENVforum sichergestellt. Es kann daher davon ausgegangen werden, dass ein grundsätzliches Interesse seitens der Regierungsmitglieder an der Arbeit des ENVforums besteht. Dass Positionen, die im ENVforum erarbeitet wurden, in den ASEM-Gipfel aufgenommen wurden, zeigen die ASEM6- und ASEM8-Gipfeltreffen. Allerdings lässt sich aufgrund der starken Informalität schwer beurteilen, wann und unter welchen Bedingungen Argumente zivilgesellschaftlicher Akteure im politischen Dialog und Entscheidungsprozess berücksichtigt werden. Es zeigt sich aber, dass diese durchaus in die politischen Diskussionen einfließen. Dies gilt auch für die globale Ebene, wie zuvor das Beispiel bzgl. UNEP gezeigt hat.

Zivilgesellschaftliche Akteure leisten einen Beitrag zur Input- bzw. demokratischen Legitimität der asiatisch-europäischen Dialogbeziehungen - allerdings auf einem eher mittleren bis niedrigen Niveau. Betrachtet man die Kriterien Inklusion, Konsultation, Transparenz, formelle Regeln, informelle Praxis und Respons, so herrscht in vielen Bereichen Verbesserungspotenzial. Die Tatsache, dass der zivilgesellschaftliche Dialog vom politischen Dialog weitestgehend entkoppelt ist, erschwert die Interaktion zwischen politischen und zivilgesellschaftlichen Akteuren. Da es auch keine formellen Regeln für diese Interaktion gibt, erscheint die Art und Weise, wie Ergebnisse des zivilgesellschaftlichen Dialogs von den politischen Eliten rezipiert werden, sehr intransparent. Die Mitgliedschaft der zivilgesellschaftlichen Akteure im ENVforum ist nur bedingt inklusiv, da politisierte und eher kritische Akteure nicht beteiligt sind. Diese haben sich als Reaktion darauf als transnationale Opposition in Form des AEPF zusammengeschlossen. 
ASEM wurde als intergouvernementales Dialogforum gegründet, um interregionale Wirtschaftsintegration zu fördern. Zivilgesellschaftliche Partizipation führte aufgrund kultureller Differenzen zu einem Kompromiss, nämlich zur Auslagerung des zivilgesellschaftlichen Dialogs in ASEF. Dieser Kompromisscharakter bleibt bis heute bestehen und auch wenn sich seit den Gründungsjahren die Interaktion zwischen politischen und zivilgesellschaftlichen Akteuren qualitativ verbessert hat, hängt zivilgesellschaftliche Partizipation auch nach wie vor noch von politischen Interessen ab. Der eher fluktuierende Einfluss zivilgesellschaftlicher Akteure auf die Ergebnisse der ASEM-Gipfel unterstützt diesen Eindruck. So leistet die Zivilgesellschaft zwar einen Beitrag zur Input- bzw. demokratischen Legitimität des ASEM-Prozesses; dessen Gewicht muss allerdings ob der herausgearbeiteten Defizite eher als gering bewertet werden.

Der vorliegende Beitrag ist nicht auf die Output-Legitimität eingegangen, die allerdings eng mit der Dimension der Input-Legitimität verknüpft ist. So müsste in weiterführenden Studien die empirische Qualität der Handlungskonsequenzen, die sich aus der interregionalen Umwelt- und Klimakooperation ergeben, untersucht werden. Dies bezieht sich nicht nur auf die aus dieser Zusammenarbeit resultierenden nationalstaatlichen Aktivitäten, wie in Kapitel 4 angedeutet, sondern auch auf die Arbeit von Stiftungen und NGOs in den ASEM-Mitgliedstaaten.

\section{Literaturverzeichnis}

Altvater, Elmar/Brunnengräber, Achim (Hrsg.), 2000: Vernetzt und verstrickt. Nichtregierungsorganisationen als gesellschaftliche Produktivkraft, Münster. $A S E F, 2011$ a: Bringing Asia and Europe together, http://www.asef.org/index.php/ about/what-we-do (Stand: 14.3.2012).

$A S E F, 2011 \mathrm{~b}$ : Asia-Europe Environment Forum: $1^{\text {st }}$ Roundtable, http:// www.asef.org/index.php/projects/themes/environment/603-asia-europe-environment-forum-1 st-roundtable-discussion (Stand: 31.8.2012).

ASEM, 2000: Asia Europe Cooperation Framework, http://www.aseminfoboard.org/rules-and-refulations/item/868-aecf-2000.html (Stand: 20.9.2012). ASEM, 2005: Dublin Agreed Principles of the Asia-Europe Foundation, http:// www.aseminfoboard.org/content/documents/Annex_1_ASEM5___Dublin_Pri nciples_of_ASEF.pdf (Stand: 20.9.2012).

ASEM, 2006 a: Chairman's Statement of the Sixth AsiaEurope Meeting, http:// www.aseminfoboard.org/summit-statement.html?limitstart $=0$ (Stand: 29.7.2013). 
ASEM, 2006 b: Chairman's statement of the sixth Asia Europe Meeting, Helsinki, 10.-11. September 2006, http://www.aseminfoboard.org/summit-statement. html (Stand: 20.9.2012).

ASEM, 2008: Chair's statement of the seventh Asia-Europe Meeting, Beijing,

24.-25. October 2008, http://www.aseminfoboard.org/summit-statement.html (Stand: 18.7.2013).

ASEM, 2010: Chair's statement of the eighth Asia Europe Meeting, Brussels, 4.-5.

October 2010, http://www.aseminfoboard.org/summit-statement.html (Stand: 18.7.2013).

ASEM, 2012: Chair's statement of the 9th Asia Europe Meeting, Vientiane, Lao

PDR, 5.-6. November 2012, http://www.aseminfoboard.org/summit-state ment.html (Stand: 25.2.2014)

Atack, Ian, 1999: Four criteria of development NGO legitimacy, in: World Development 27 (5), 855-864.

Auer, M. R., 2000: Who participates in global environmental governance? Partial answers from international relations theory, in: Policy Sciences 33 (2), 155-180.

Bäckstrand, Karin/Khan, Jamil/Kronsell, Annica/Lövbrand, Eva (Hrsg.), 2010 a: Environmental politics and deliberative democracy. Examining the promise of new modes of governance, Cheltenham.

Bäckstrand, Karin/Khan, Jamil/Kronsell, Annica/Lövbrand, Eva, 2010 b: The promise of new modes of environmental governance, in: Karin Bäckstrand/Jamil Khan/Annica Kronsell/Eva Lövbrand (Hrsg.), Environmental politics and deliberative democracy. Examining the promise of new modes of governance, Cheltenham, 3-27.

Beisheim, Marianne, 2005: NGOs und die (politische) Frage nach ihrer Legitimation. Das Beispiel Klimapolitik, in: Achim Brunnengräber/Ansgar Klein/Heike Walk (Hrsg.), NGOs im Prozess der Globalisierung. Mächtige Zwerge, umstrittene Riesen, Wiesbaden, 242-265.

Bernstein, Steven, 2004: Legitimacy in Global Environmental Governance, in: Journal of International Law and International Relations 1 (Winter 2004/Spring 2005), 139-166.

Bersick, Sebastian, 2008: The democratization of inter- and transregional dialogues. The role of civil society, NGO and parliamentarians, in: Jürgen Rüland (Hrsg.), Asia-Europe relations. Building block or stumbling block for global governance?, London, 244-270.

Beyer, Corneli, 2007: Non-Governmental Organisations as motors of change, in: Government and Opposition 42 (4), 513-535. 
Biermann, Frank/Pattberg, Philipp/van Asselt, Harro/Zelli, Fariborz, 2009: The fragmentation of global governance architectures. A framework for analysis, in: Global Environmental Politics 9 (4), 14-40.

Brand, Ulrich, 2005: Gegen-Hegemonie. Perspektiven globalisierungskritischer Strategien, Hamburg.

Carrapatoso, Astrid, 2011: Climate policy diffusion. Interregional dialogue in China-EU Relations, in: Global Change, Peace \& Security 23 (2), 177-194.

Dent, Christopher, 2004: The Asia-Europe Meeting and Inter-Regionalism. Toward a theory of multilateral utility, in: Asian Survey 44 (2), 213-236.

Dingwerth, Klaus, 2007: The new transnationalism. Transnational governance and democratic legitimacy, Basingstoke.

Doherty, Brian, 2002: Ideas and Actions in the Green Movement, London.

Dryzek, John S., 2000: Deliberative democracy and beyond. Liberals, critics, contestations, New York.

Fort, Bertrand (Hrsg.), 2004: Reinforcing Asia-Europe Co-operation on Climate Change, Singapur.

Fort, Bertrand, 2005: Overview on ASEM co-operation in the field of the environment and introduction to the Asia-Europe Environment Forum, in: Ramon Molina (Hrsg.), Asia-Europe co-operation on the environment. Towards sustainable forest management, Singapur.

Fort, Bertrand/Iglesias, Sol (Hrsg.), 2006: The Jakarta 12 Asia-Europe Agendas for Sustainable Development, Singapur.

Frantz, Christiane/Martens, Kerstin, 2006: Nichtregierungsorganisationen (NGOs), Wiesbaden.

Gaens, Bart, 2008 a: ASEM's background and rationale, in: Bart Gaens (Hrsg.), Europe-Asia interregional relations. A decade of ASEM, Aldershot, 9-28.

Gaens, Bart, 2008 b: ASEM as an economy-oriented partnership, in: Bart Gaens (Hrsg.), Europe-Asia interregional relations. A decade of ASEM, Aldershot, 29-48.

Gilson, Julie, 2002: Asia meets Europe. Inter-regionalism and the Asia-Europe Meeting, Cheltenham/Northampton.

Gilson, Julie, 2005: New Interregionalism? The EU and East Asia, in: European Integration 27 (3), 307-326.

Grugel, Jean, 2004: New regionalism and modes of governance. Comparing US and EU strategies in Latin America, in: European Journal of International Relations 10 (4), 603-626. 
Hänggi, Heiner, 2006: Interregionalism as a multifaceted phenomenon, in: Heiner Hänggi/Ralf Roloff/Jürgen Rüland (Hrsg.), Interregionalism and international relations. A stepping stone to global governance?, London, 31-62.

Hänggi, Heiner/Roloff, Ralf/Rüland, Jürgen, 2006: Interregionalism. A new phenomenon in international relations, in: Hänggi, Heiner/Roloff, Ralf/Rüland, Jürgen (Hrsg.), Interregionalism and International Relations. A stepping stone to global governance?, London, 3-14.

Hwee, Yeo Lay, 2007: The Inter-regional Dimension of EU-Asia Relations. EUAsean and the Asia-Europe Meeting (ASEM) Process, in: European Studies 25 (1), 173-191.

Keohane, Robert O./Macedo, Stephen/Moravcsik, Andrew, 2009: Democracy-enhancing multilateralism, in: International Organization 63, 1-31.

Keva, Silja, 2008: ASEM and civil society, in: Bart Gaens (Hrsg.), Europe-Asia Interregional Relations, Aldershot, Ashgate, 101-114.

Kronsell, Annica/Bäckstrand, Karin, 2010: Rationalities and forms of governance. a framework for analysing the legitimacy of new modes of governance, in: Karin Backstrand/Jamil Khan/Annica Kronsell/Eva Lövbrand (Hrsg.), Environmental politics and deliberative democracy, Cheltenham, 28-46.

Manea, Gabriela, 2008: Human rights and the interregional dialogue between Asia and Europe: ASEAN-EU relations and ASEM, in: The Pacific Review 21 (3), 369-396.

Mansfield, Edward D./Pevehouse, Jon C., 2006: Democratization and international organizations, in: International Organization 60, 137-167.

Maull, Hanns W., 2010: Das Asia-Europe Meeting (ASEM). Baustein effektiverer globaler Ordnungsstrukturen?, in: Dirk Nabers (Hrsg.), Multilaterale Institutionen in Ostasien-Pazifik, Wiesbaden, 181-206.

Molina, Ramon (Hrsg.), 2005: Asia-Europe Co-operation on the Environment: Towards Sustainable Forest Management, Singapur.

Oberthür, Sebastian/Stokke, Olav Schram, 2010: Introduction: Institutional interaction in global environmental change, in: Sebastian Oberthür/Olav Schram Stokke (Hrsg.), Managing institutional complexity. Regime interplay and global environmental change, Cambridge, 1-24.

Paget, Roger/Blouch, Raleigh A./Juliani, S./Sutton, Richard, 2005: Evaluation of the Indonesian Biodiversity Foundation (IBF) Project - KEHATI, http:// www.oecd.org/derec/unitedstates/35890837.pdf (Stand: 4.9.2012).

Pevehouse, Jon C., 2002: Democracy from the outside-in? International Organizations and democratization, in: International Organization 56 (3), 515-549. 
Robles, Alfredo, 2008: The Asia-Europe Meeting. The theory and practice of Interregionalism, London.

Roloff, Ralf, 2006: Interregionalism in theoretical perspective: state of the art, in: Heiner Hänggi/Ralf Roloff/Jürgen Rüland (Hrsg.), Interregionalism and international relations, London/New York, 17-30.

Rüland, Jürgen, 2002: The European Union as an inter- and transregional actor. Lessons for global governance from Europe's relation's with Asia, Canberra.

Rüland, Jürgen, 2006: Interregionalism. An unfinished agenda, in: Heiner Hänggi/ Ralf Roloff/Jürgen Rüland (Hrsg.), Interregionalism and International Relations, London, 295-313.

Rüland, Jürgen, 2010: Balancers, multilateral utilities or regional identity builders? International Relations and the study of Interregionalism, in: Journal of European Public Policy 17 (8), 1271-1283.

Rüland, Jürgen, 2012: The rise of "diminished multilateralism". East Asian and European forum shopping in Global Governance, in: Asia-Europe Journal 9 (2-4), 255-270.

Rüland, Jürgen, 2014: Interregionalism and International Relations: Reanimating an obsolescent research agenda?, in: Francis Baert/Tiziana Scaramagli/Fredrik Söderbaum (Hrsg.), Intersecting Interregionalism, Heidelberg, 15-35.

Rüland, Jürgen/Schubert, Gunter/Schucher, Gunter/Storz, Cornelia, 2008: AsianEuropean Relations. Building Blocks for Global Governance?, Abingdon/New York.

Scharpf, Fritz Wilhelm, 1999: Regieren in Europa. Effektiv und demokratisch?, Frankfurt a. M.

Schmidt, Vivien A., 2010: Taking ideas and discourse seriously. Explaining change through discursive institutionalism as the fourth new institutionalism, in: European Political Science Review 2 (1), 1-25.

Steffek, Jens/Bendrath, Ralf/Dalferth, Simon/Hahn, Kristina/Piewitt, Martina/Rodekamp, Meike, 2010: Assessing the democratic legitimacy of transnational CSOs: Five criteria, in: Jens Steffek/Kristina Hahn (Hrsg.), Evaluating transnational NGOs. Legitimacy, accountability, representation, Basingstoke, 100-125. Steffek, Jens/Hahn, Kristina, 2010: Introduction: Transnational NGOs and legitimacy, accountability, representation, in: Jens Steffek/Kristina Hahn (Hrsg.), Evaluating transnational NGOs. Legitimacy, accountability, representation, Basingstoke, 1-25.

Steffek, Jens/Nanz, Patrizia/Kissling, Claudia, 2008: Emergent Patterns of Civil Society Participation in Global and European Governance, in: Jens Steffek/Pa- 
trizia Nanz/Claudia Kissling (Hrsg.), Civil Society Participation in European and Global Governance, Basingstoke, 1-24.

University of Helsinki Network for European Studies, 2006: ASEM in its Tenth Year Looking Back, Looking Forward. An evaluation of ASEM in its first decade and an exploration of its future possibilities, Helsinki, http://www.mofa.go.jp/policy/ economy/asem/tenth/report1.pdf (Stand: 5.2.2014).

Williams, Marc, 2005: Globalization and civil society. in: John Ravenhill (Hrsg.), Global political economy, Oxford/New York, 344-369.

Zajak, Sabrina, 2012: Interregional governance and transnational civil societal opposition: The Asia-Europe Meeting and its counter-mobilization. Paper presented at the "Kongress der Deutschen Vereinigung für Politische Wissenschaft", 24.-28. September 2012 in Tübingen.

Zelli, Fariborz, 2011: The fragmentation of the global climate governance architecture, in: WIREs Climatic Change 2 (2), 255-270.

Korrespondenzanschrift:

Dr. Astrid Carrapatoso

Seminar für Wissenschaftliche Politik

Rempartstr. 15

79085 Freiburg

E-Mail: astrid.carrapatoso@politik.uni-freiburg.de 